\title{
Cortisol plasmático como indicador de estresse em colopexias laparoscópicas com implante de tela de polipropileno em cães
}

\author{
Serum cortisol to assess stress associated with laparoscopic colopexy \\ using polypropylene mesh in dogs

\begin{abstract}
Ricardo Zanella', Guilherme Thomazi², Nédio Guizzo Junior ${ }^{3}$, Julio David Spagnolo4, Luciana Dambrósio Guimarães ${ }^{3} \&$ Maurício Veloso Brun ${ }^{3}$
\end{abstract}

\begin{abstract}
RESUMO
A realização de cirurgias convencionais, mesmo com a utilização recomendada de fármacos analgésicos, geralmente está associada a um período de recuperação longo, além de causar estresse, dor e desconforto nos animais, dependendo do grau de invasão do procedimento. Considerando a crescente busca por métodos que minimizem o estresse cirúrgico em cães, reduzindo o período de recuperação pós-cirúrgico, o presente trabalho foi desenvolvido para avaliar a técnica de colopexia laparoscópica em relação aos níveis de cortisol plasmático, as frequências respiratórias, e as frequências cardíacas nos períodos pré, trans e pós-operatório imediatos. Foram utilizados nove cães, fêmeas adultas, SRD. As coletas sanguíneas para mensuração dos níveis de cortisol, e os parâmetros de frequências respiratórias (FR) e cardíacas (FC) foram efetuadas a partir do préoperatório até o término do procedimento. Após esse período, a tomada de dados foi realizada de três em três horas até completar as primeiras 12 horas pós-operatórias. Os níveis de cortisol foram mensurados com a utilização de Kit ELISA para cortisol. Os resultados foram submetidos à ANOVA e ao teste de Tukey, considerando as diferenças significativas $p \leq 0,05$. Aumentos dos níveis de cortisol, FR e FC foram verificados principalmente no período após tricotomia, mantendo-se constantes no transcirúrgico, com um novo aumento no pós-operatório imediato, no período de recuperação anestésica. Tais parâmetros retornaram aos valores basais nove horas após o término da operação. Conclui-se que a colopexia laparoscópica causa estresse ao paciente, contudo os valores de cortisol plasmático apresentaram-se dentro de valores fisiológicos para a espécie em estudo e tenderam ao retorno às condições basais, nove horas após a operação.
\end{abstract}

Descritores: cirurgia, estresse, colopexia laparoscópica, cortisol, caninos.

\begin{abstract}
The realization of conventional surgical procedures, even using the recommended doses of analgesics drugs, generally are associated with a long recovery period, post-surgical trauma, stress and pain in animals. That can vary according with the procedure, extension and time of the surgery. Considering the increased interest in procedures that minimize surgical stress and the recovery period after the surgical procedure in dogs, this present work was developed to evaluate a new technique of laparoscopic colopexy with the alterations of cortisol levels, heart rate and respiratory frequencies in dogs. For this study, nine female dogs were submitted to a colopexy. Blood samples and data of heart rate and respiratory frequency were sampled in intervals of 10min. from the preoperative period until the end of the procedure. After, these parameters were assessed every three hours for 12 hours. Cortisol levels were measured using an ELISA Kit for cortisol. Results were analyzed by ANOVA and comparisons were made using Tukey's test. Significance levels was set at $p \leq 0.05$. The increase of the heart rate, respiratory frequency and cortisol levels were mainly observed in the period after shaving and after the end of the anesthesia. Values were kept stable during all the surgery, and coming to the basal conditions after nine hours in the post-operative period. The laparoscopic colopexy is a stress-associated surgery, but it produces lower increasing of cortisol levels returning to normal in 9 hours after the surgery.
\end{abstract}

Keywords: surgery, stress, laparoscopic colopexy, cortisol, canine.

${ }^{1}$ Department of Animal Sciences, Washington State University (WSU), Pullman, WA, EUA. ${ }^{2}$ Universidade de Caxias do Sul (UCS), Caxias do Sul, RS, Brasil. ${ }^{3}$ Curso de Medicina Veterinária (FAMV), Universidade de Passo Fundo (UPF), Campus I, BR 285, Km 171, Bairro São José, C.P. 611/631, CEP 99001-970 Passo Fundo, RS, Brasil. ${ }^{4}$ Universidade de São Paulo (USP), São Paulo, SP, Brasil. CORRESPONDÊNCIA: M.V. Brun [mbrun@upf.br ; Fax: +55 (54) 3316 8487]. 


\section{INTRODUÇÃO}

O uso de intervenções cirúrgicas por via laparoscópica vem crescendo em Medicina Veterinária [26]. Esse procedimento minimiza o estresse $\mathrm{e}$ a dor pós-operatória, apresenta melhores resultados estéticos, possibilita recuperação mais rápida e melhor visualização dos órgãos intracavitários, tal como tem sido relatado em humanos [17]. Atualmente, são realizados diferentes procedimentos laparoscópicos na rotina de pequenos animais, que incluem biópsias de diferentes órgãos [14,25], orquiectomia [28], ovariectomia [1], cistotomia [32], colopexia [7,8], cistopexia [30], gastropexia [18,31], nefrectomia [6,32] e ovário-histerectomia [34]. Confrontando operações em campo aberto (convencionais) e laparoscópicas, verificou-se que quanto maior o grau de invasão do procedimento, maior será o estresse causado ao animal e, consequentemente, o seu período de recuperação $[24,33]$. Dentre as cirurgias relatadas, a colopexia é indicada em cães no tratamento de prolapso retal recidivante não responsivo à sutura de retenção em bolsa-de-tabaco e também como procedimento complementar no tratamento de hérnias perianais $[10,11,29]$. $\mathrm{Na}$ busca por novas técnicas minimamente invasivas e observando os resultados experimentais associados à colopexia laparoscópica com retalho de tela de polipropileno [9], justificando assim o aprofundamento científico imposto por tal procedimento, destacandose o estresse cirúrgico. $\mathrm{O}$ trabalho objetivou avaliar o estresse cirúrgico através da mensuração dos níveis de cortisol plasmático, frequências cardíaca (FC) e respiratória (FR) nos períodos trans e pós-operatórios em cães experimentalmente submetidos à colopexia com tela de polipropileno por videocirurgia.

\section{MATERIAS E MÉTODOS}

\section{Animais}

Foram utilizados nove cães, fêmeas adultas, hígidas, com peso médio de $6,28+1,92 \mathrm{~kg}$, provenientes do Biotério da Universidade de Passo Fundo. Durante todo o experimento, foram mantidas em gaiolas individualizadas, com alimentação sólida (ração industrial) e água ad libitum. Todos os animais passaram por período de ambientação nas instalações, onde seriam posteriormente mantidos. Previamente às operações, os cães foram submetidos à avaliação clínica e coleta de amostra sanguínea para a avaliação do hemograma e proteínas plasmáticas. Verificado que tais parâmetros encontravam-se dentro dos valores e das condições normais para a espécie, os pacientes foram encaminhados para a cirurgia.

\section{Procedimento cirúrgico}

Os animais foram pré-medicados com a aplicação de $0,05 \mathrm{mg} \cdot \mathrm{kg}^{-1}$ de maleato de acetilpromazina ${ }^{1}$ e de $0,5 \mathrm{mg} \cdot \mathrm{kg}^{-1}$ de sulfato de morfina ${ }^{2}$ associados na mesma seringa e aplicados por via intramuscular e, então, procedeu-se ampla tricotomia abdominal e cervical ventral. Os pacientes foram induzidos com $5 \mathrm{mg} . \mathrm{kg}^{-1}$ de propofol $^{3}$ a partir do cateter aplicado na veia cefálica. A manutenção foi obtida com vaporização de 0,5 a $1,0 \mathrm{~V} \%$ de

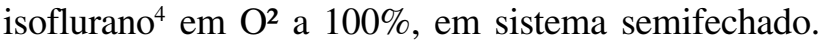
Todas as operações foram realizadas por um único cirurgião com experiência em videolaparoscopia, para minimizar possíveis alterações decorrentes de diferentes graus de manipulação tecidual. A técnica cirúrgica realizada constou da produção de pneumo-peritônio com $\mathrm{CO}_{2}(12 \mathrm{mmHg})$ pelo método aberto. Posteriormente, foram aplicados três portais, um de $10 \mathrm{~mm}$, na linha média ventral, caudalmente à cicatriz umbilical, e outros dois nas paredes abdominais direita $(10 \mathrm{~mm})$ e esquerda (5mm). Após a eleição do sítio de colopexia, foram realizadas duas incisões paralelas no músculo transverso abdominal esquerdo, formando-se um flape bipedicular. Procedeu-se a dissecação do mesocólon, que permitiu a passagem de uma tira de tela de polipropileno $(12 \times 1,5 \mathrm{~cm})$ através deste e sob o flape. As duas extremidades do implante foram unidas com clipe de titânio, colocando em contato o órgão com a parede abdominal. As feridas foram ocluídas em nível muscular e/ou cutâneo, na dependência de suas dimensões. Os animais receberam como medicação analgésica pósoperatória $2 \mathrm{mg} . \mathrm{kg}^{-1}$ de Ketoprofeno ${ }^{5}$, SC, SID, durante três dias, e as feridas operatórias foram diariamente higienizadas com solução de $\mathrm{NaCl}$ 0,9\% (TID, por sete dias).

\section{Coleta de sangue, frequências respiratória e cardíaca}

As coletas de sangue foram feitas na veia jugular, sendo que, após a indução anestésica, aplicou-se um cateter intravenoso (n. 20) nesse vaso, para facilitar a coleta sanguínea. Coletou-se a primeira amostra de sangue para a determinação dos valores basais de cortisol plasmático, anteriormente à aplicação da pré-medicação anestésica. A partir de então, coletas seriadas, a cada 10 minutos, foram realizadas, até a sutura de pele. 
Da mesma forma, avaliaram-se as frequências cardíacas (FC) e respiratórias (FR) nos mesmos intervalos de tempo. Posteriormente, as coletas de sangue e a quantificação das frequências supracitadas foram executadas a cada três horas nas primeiras 12 horas do período pós-operatório. Os tempos das coletas seguiram os seguintes períodos: $\mathrm{A}=$ pré-tricotomia e anteriormente à pré-medicação (valor basal); $\mathrm{B}=$ pós-tricotomia e após aplicação da medicação pré-anestésica; $\mathrm{C}=$ pré-indução anestésica; $\mathrm{D}=$ pós-indução anestésica; $\mathrm{E}=$ período transoperatório e de manutenção da anestesia (valores médios); $\mathrm{F}=$ final da colopexia e desinsu-flação da cavidade; $\mathrm{G}=$ =clusão da parede abdominal; $3 \mathrm{~h}=$ três horas de pós-operatório; $6 \mathrm{~h}=$ seis horas de pós-operatório; $9 \mathrm{~h}=$ nove horas de pós-operatório; $12 \mathrm{~h}=12$ horas de pós-operatório.

\section{Análise do cortisol}

As amostras sanguíneas foram armazenadas em tubos de vidro e centrifugadas a $2000 \mathrm{x} \mathrm{g}$ (EPPENDORF $5804 \mathrm{R}$ ) por 15 minutos, num período inferior a 30 minutos da coleta. Após o congelamento a $-196^{\circ} \mathrm{C}$, a determinação do cortisol foi feita por radioimunoensaio, através de um kit comercial para ELISA (IBL - Immuno Biological Laboratories).

\section{Análise estatística}

Os resultados foram analisados utilizando o programa PROC MIX, SAS repeated measurement regression $(\mathrm{SCH})$, para verificar o efeito do tempo nas medidas fisiológicas de cortisol sanguíneo, batimentos cardíacos e frequência respiratória. Os valores médios de todos os parâmetros avaliados foram submetidos à ANOVA seguidos do teste de Tukey, considerando as diferenças significativas quando $p \leq 0,05$.

\section{RESULTADOS}

As cirurgias foram realizadas no tempo total de 37,00ะ14,49min, permitindo um número médio de 12,3 coletas sanguíneas. As amostras obtidas durante o período cirúrgico foram agrupadas, analisando-se somente o valor médio, uma vez que não foi possível correlacionar a obtenção sanguínea com a etapa ou manobra da operação. Na Tabela 1, encontram-se os valores referentes à $\mathrm{FR}, \mathrm{FC}$ e níveis séricos de cortisol durantes as coletas. Observou-se um aumento significativo $(p<0.05)$ nos batimentos cardíacos dos animais, frequência respiratória e nos níveis de cortisol sanguíneo no momento cirúrgico e de recuperação dos animais. Ficou evidenciado a ocorrência de um aumento significativo do

Tabela 1. Variações nos níveis de cortisol plasmático $(\mu \mathrm{g} / \mathrm{dl})$, frequência respiratória (FR) e frequência cardíaca (FC) em cães submetidos à colopexia laparoscópica com implante de tela de polipropileno. As coletas foram procedidas nos períodos correspondendo a: contato com o paciente previamente a realização da tricotomia (A); pós-tricotomia e pós-aplicação dos fármacos pré-anestésicos (B); pré-indução anestésica (C); pós-indução anestésica (D); período transoperatório e de manutenção do plano anestésico (E); final da colopexia e desinsuflação da cavidade (F); oclusão da ferida operatória $(\mathrm{G}) ; 3 \mathrm{~h}$; 6h; $9 \mathrm{~h}$; e 12h após o término da operação.

\begin{tabular}{cccc}
\hline Coletas & Cortisol & FR & FC \\
\hline A & $0.81 \pm 0.31$ & $25 \pm 6.6$ & $99.2 \pm 18$ \\
B & $1.61 \pm 0.43 * *$ & $31.3 \pm 3.01 *$ & $107.3 \pm 23$ \\
C & $1.63 \pm 0.48 * *$ & $19.33 \pm 5.89 * *$ & $141 \pm 57 *$ \\
D & $1.2 \pm 0.62$ & $17.33 \pm 11.78$ & $108.5 \pm 11.41$ \\
E & $1.42 \pm 0.83 *$ & $12.7 \pm 5.03 * *$ & $118 \pm 18 *$ \\
F & $1.59 \pm 0.55 *$ & $17.67 \pm 4.4$ & $117.5 \pm 11.8 *$ \\
G & $2.76 \pm 0.34 *$ & $18 \pm 2.8$ & $133.5 \pm 12 *$ \\
$3 \mathrm{~h}$ & $1.34 \pm 0.78$ & $19.17 \pm 6.52$ & $106 \pm 10.2 *$ \\
$6 \mathrm{~h}$ & $2.16 \pm 0.76 * *$ & $19.33 \pm 2.73$ & $117 \pm 13 *$ \\
$9 \mathrm{~h}$ & $1.02 \pm 0.6$ & $20.8 \pm 3.35$ & $129 \pm 25 *$ \\
$12 \mathrm{~h}$ & $1.72 \pm 0.5 *$ & $20 \pm 4.62$ & $132 \pm 19.8 *$ \\
\hline
\end{tabular}

$*$ p-value $<0.05 * *$ p-value $<0.002$ 


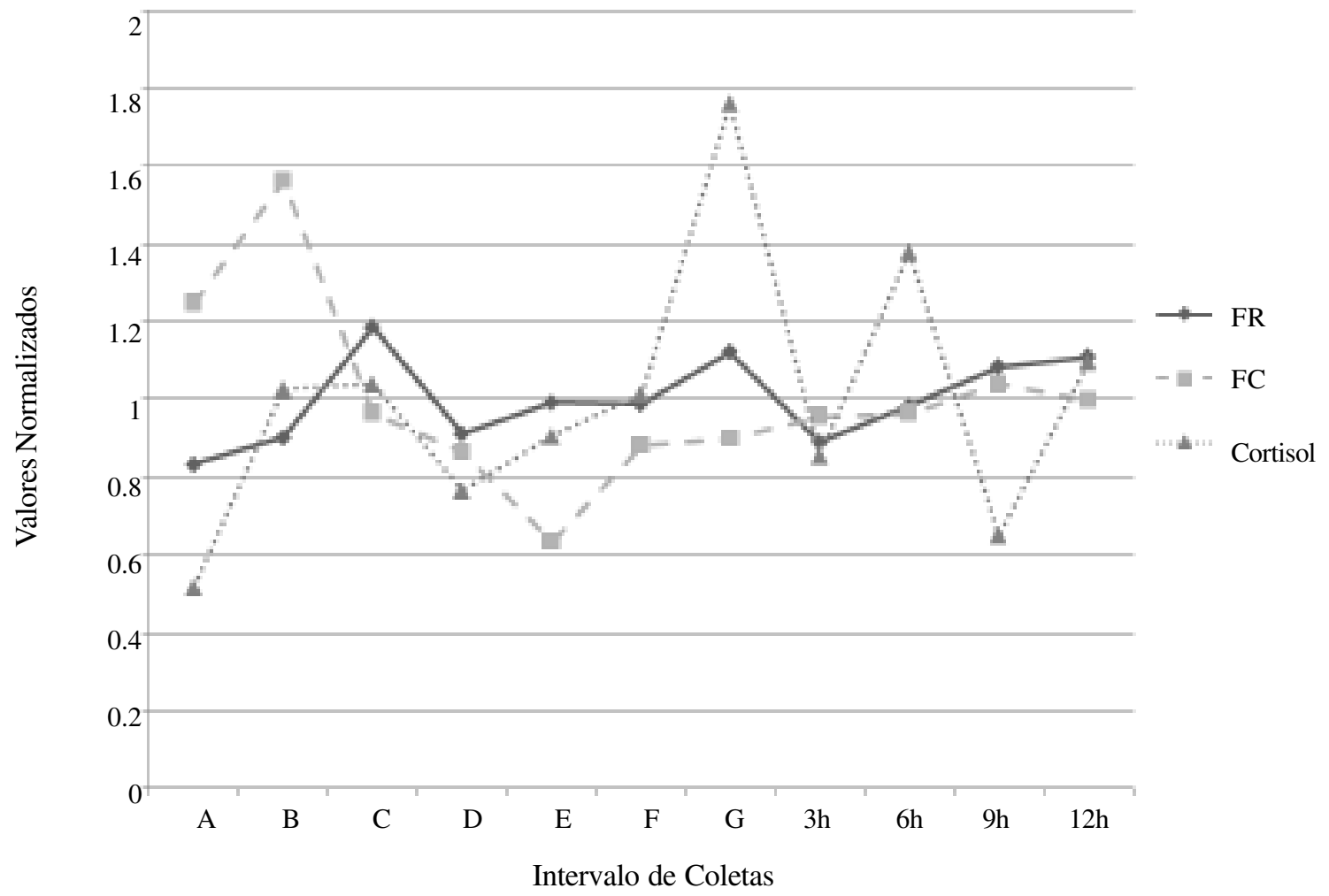

Figura 1. Comparação entre as médias normalizadas dos níveis de Cortisol Plasmático, Frequência Cardíaca (FC) e Frequência Respiratória (FR) nos diferentes pontos de coletas. A normalização dos valores foi realizada através da divisão de cada ponto de coleta individual pela média geral dos parâmetros de interesse. A normalização dos valores foi desenvolvida para uma melhor visualização gráfica da relação entre as mudanças ocorridas nos valores dos três parâmetros.

cortisol pela simples submissão à tricotomia antes mesmo de qualquer aplicação medicamentosa. Os níveis de cortisol plasmático (Tabela 1, Figura 1) demonstraram aumento significativo no período subsequente à tricotomia, até a pré-indução, tendo um decréscimo não significativo no período pós-indução, mantendo os seus níveis estabilizados durante o procedimento $(p=0.09)$. Houve uma significativa elevação dos níveis de cortisol no período da sutura $(\mathrm{G})(p<0.01)$ e às seis horas após a cirurgia $(6 \mathrm{~h})(p<0.002)$, sendo que nove horas após a cirurgia os níveis retornaram para os valores basais.

Em relação aos valores de frequência respiratória (FR) (Tabela 1, Figura 1), observou-se que no período posterior à tricotomia (B), ocorreu aumento significativo $(p<0.05)$, quando comparado com o período de repouso. Além disso, após a administração da medicação pré-anestésica, ocorreu um decréscimo desse parâmetro $(p<0.001)$. No período correspondente ao procedimento cirúrgico (E), os valores da FR foram significativamente inferiores quando comparados com os valores basais $(p=0.002)$, mantendo-se na média em 12,72 mr/min. Quando deu-se início à oclusão da ferida operatória, ocorreu um aumento não significativo desse parâmetro. Após isso, a FR se manteve sem diferenças significativas até o período de 12 horas após a cirurgia.

A frequência cardíaca demonstrou aumento significativo no período de pré-indução anestésica (C) quando comparado com o período de repouso dos animais (A) $(p<0.05)$. Os valores de batimentos cardíacos no período pré-tricotomia não foram significativamente diferentes ao período de pós-tricotomia. Contudo, houve decréscimo não significativo nos valores desse parâmetro após a aplicação da medicação pré-anestésica $(p=0.09)$. Posteriormente, a frequência cardíaca manteve-se constante por todo o transoperatório, porém significativamente mais elevada do que o período de repouso dos animais $(p=0.04)$. No período correspondente ao final da cirurgia e ao inicio da oclusão da ferida, ocorreu um aumento significativo nos batimentos cardíacos dos animais $(p<0.05)$.

\section{DISCUSSÃO}

Vários parâmetros fisiológicos têm sido utilizados como indicadores de bem-estar animal, incluindo catecolaminas, funções imunes, batimentos cardíacos 
e frequência respiratória [4,37], mas, recentemente, pesquisas em cães têm demonstrado especial atenção para os glucocorticóides, especificamente cortisol [4,5, 19,20]. Os níveis de cortisol podem ser mensurados no plasma, porém existem outros métodos menos invasivos. A dosagem de cortisol livre na urina apresenta como vantagens teóricas o fato de ser imune a flutuações da proteína carregadora de cortisol (CBG). Por outro lado, a dosagem do cortisol livre urinário apresenta uma limitação específica que concerne à coleta da urina, procedimento que exige participação ativa do paciente. Métodos mais específicos, como a espectrometria de massa é a que apresenta maior potencial, embora o custo elevado seja a maior barreira [36]. A concentração de cortisol plasmático tem sido muito utilizada para medir dor induzida e estresse em cães após cirurgias [16,23]. A mensuração dos níveis de cortisol é comumente usada em pesquisa, mas não como um método clínico de avaliação [16].

Diferentemente de outros estudos $[12,13,15$, 17,27], no presente trabalho, procurou-se coletar amostras sanguíneas em intervalos curtos de 10 minutos, considerando que o pico de cortisol em cães tende a ocorrer de 15 a 30 minutos após o estímulo [21,27]. Assim, ao contrário do que se descreve em outros trabalhos [17,27], os autores acreditam que os valores basais devem ser estimados o mais próximo possível do primeiro contato pré-cirúrgico com o paciente, para que não sejam contabilizados os picos hormonais decorrentes da manipulação do paciente pelo avaliador justificando o maior pico da FR no momento póstricotomia (Figura 1) Considera-se que a utilização de fármacos narcóticos, tal como o sulfato de morfina empregado no presente trabalho como pré-medicação, pode suprimir a liberação de corticosteróides [27], o que explicaria os valores mais baixos no tempo D (Figura 1) em relação às duas avaliações imediatamente posteriores. Futuras análises devem ser realizadas para a comprovação do efeito de fármacos narcóticos com a liberação de cortisol em cães. Considera-se que no momento D (imediatamente após a indução), importantes alterações de cortisol referentes à aplicação anestésica ainda não seriam contabilizadas pelo tempo necessário para o pico hormonal atribuindo-se então tais alterações hormonais ao uso do opióide [21]. Como o momento de oclusão das feridas operatórias ocorreu simultaneamente à suspensão da medicação anestésica, confirma-se a ocorrência de maior liberação desse hormônio durante o período anestésico (Figura 1).
O retorno da etapa de inconsciência e os efeitos da ausência de anestesia ocasionam a elevação de cortisol plasmático, devido aos efeitos dos estímulos nocioceptivos residuais e da resposta cognitiva contextual [13,27]. A retomada de valores elevados do hormônio estudado às seis horas do pós-operatório pode ter ocorrido por analgesia insuficiente, uma vez que foi administrado anti-inflamatório não esteróide unicamente no pós-operatório, corroborando com outros autores [21]. Considera-se que nesse período já haveria supressão da condição analgésica máxima do sulfato de morfina, e que os efeitos da irritação diafragmática e peritoneal ocasionada pelo $\mathrm{CO}_{2}$ ainda estariam presentes [2]. Esses eventos poderiam explicar o maior tempo necessário de retomada aos valores basais de cortisol no presente estudo (que ocorreu no período de nove horas de pós-operatório) em relação aos dados observados por outros autores, os quais demonstram que o hormônio alcançou os níveis préoperatórios de quatro a seis horas após a cirurgia. Por outro lado, coletaram sangue para a mensuração dos valores basais, imediatamente após o contato com o animal, considera-se que nesse momento os animais demonstraram valores de cortisol baixos devido ao curto intervalo entre manipulação e coleta, tempo esse consideravelmente inferior ao descrito na literatura como necessário para a obtenção de picos desse hormônio [27]. Ao se trabalhar com valores mais baixos, no presente trabalho, do que o relatado previamente, também se deve considerar que poderia ser necessário maior tempo para a retomada deste. Assim como o descrito em OSH laparoscópica ou convencional [13,17] e nefrectomias [27], a elevação em relação aos valores basais registrada durante o trans e o pós-operatório demonstra que a cirurgia laparoscópica, assim como a aberta, ocasiona estresse em cães. $\mathrm{O}$ fator principal para a elevação desse hormônio pode ser a realização do pneumoperitônio [27], conforme resultados experimentais e os observados em outros estudos [22]. Considerando-se os dados desse trabalho, acredita-se ainda que o efeito da manipulação tecidual e as lesões teciduais transoperatórias também possam ter influenciado diretamente na elevação dos valores hormonais.

O cortisol plasmático parece ser um bom indicador de estresse cirúrgico no modelo canino, e o rápido declínio desse hormônio após laparoscópias em relação à aberta, pode indicar um menor grau, ou mais rápida resolução do estresse cirúrgico. No presente 
estudo, apesar de não haver comparação com procedimento similar por celiotomia, acredita-se que a técnica estudada ocasiona pouco estresse aos pacientes, pois os resultados pré, trans e pós-operatórios demonstraram baixos níveis de cortisol, com maior pico no momento da oclusão da ferida operatória (Figura 1), condição esperada conforme estudos prévios [27].

\section{CONCLUSÕES}

Nas condições do presente estudo, conclui-se que a colopexia laparoscópica com tela de polipropileno em cães está associada a estresse trans e pós-operatório imediato dos pacientes. Contudo a elevação do cortisol plasmático é discreta, e os valores desse hormônio tendem a retornar à condição basal dentro das primeiras nove horas do pós-operatório.

\section{NOTAS INFORMATIVAS}

${ }^{1}$ ACEPRAN - Univet S/A, São Paulo, SP, Brasil. ${ }^{2}$ DIMORF - Lab. Cristália, Itapira, SP, Brasil. ${ }^{3}$ FRESOFOL - Fresenius Kabi, Campinas, SP, Brasil. ${ }^{4}$ ISOFORINE - Cristália P.Q.F., Itapira, SP, Brasil. ${ }^{5}$ KETOFEN - Merial, Campinas, SP, Brasil.

\section{REFERÊNCIAS}

1 Beck C.A.C., Pippi N.L., Raiser A.G., Brun M.V., Gonçalves G.F., Portella L.C.V., Leme M.C. \& Stedile R. 2004. Ovariectomia laparoscópica em uma cadela com ovários remanescentes: relato de caso. MEDVEP. Revista Cientifica de Medicina Veterinária. 2: 15-19.

2 Beede D.S., Mcnevin R.P., Crain J.M., Letourneau J.G., Belani K.G.,Abrams J.A. \& Goodale R.L. 1993. Evidence for venous stasis after abdominal insufflation for laparoscopic cholecystectomy. Surgery, gynecology \& obstetrics. 176: 444-447.

3 Beerda B., Schilder M.B.H., Janssen N.S. \& Mol J.A. 1996. The use of saliva cortisol, urinary cortisol, and catecholamine measurements for a noninvasive assessment of stress responses in dogs. Hormones and Behavior. 30: 272-279.

4 Beerda B., Schilder M.B., Bernadina W., van Hooff J.A., de Vries H.W. \& Mol J.A. 1999. Chronic stress in dogs subjected to social and spatial restriction. II. Hormonal and immunological responses. Physiology \& Behavior. 66: 243-254.

5 Bergeron R., Scott S.L., Émond J.P., Mercier F., Cook N.J. \& Schaefer A.L. 2002. Physiology and behavior of dogs during air transport. Canadian Journal of Veterinary Research. 66: 211-216.

6 Brun M.V., Beck C.A., Mariano M.B., Antunes R. \& Pigatto J.A.T. 2002. Nefrectomia laparoscópica em cão parasitado por Dioctophyma renale - relato de caso. Arquivos de Ciências Veterinárias e Zoologia da UNIPAR. 5: 145-152.

7 Brun M.V., Pippi N.L., Beck C.A.C., Contesini E.A., Pereira R.A., Stedile R., Bonfada A.T., Columé L.M., Gomes K., Vieira Junior A.R.P. \& Silva T.F. 2004. Colopexia incisional por celiotomia ou transparietal auxiliada por laparoscopia em cães. Ciência Rural. 34: 829-837.

8 Brun M.V., Pippi N.L., Beck C.A.C., Contesini E.A., Chaves E., Pereira R.A., Stedile R., Gomes K., Schettini B.R., Rocha F., Bonfada A.T., Columé L.M. \& Vieira Junior A.R.P. 2004. Resistência à tração de colopexias incisionais realizadas por cirurgia laparoscópica ou celiotomia em cães. Ciência Rural. 34: 839-845.

9 Brun M.V., Guimarães L.D., Barcelos H.H.A., Guizzo Junior N. \& Pereira R.A. 2007. Colopexia laparoscópica com retalho de tela de polipropileno. Arquivo Brasileiro de Medicina Veterinária e Zootecnia. 59:119-126.

10 Clarke R.E. 1983. Perineal hernia in the dog: a modified technique of repair. Australian Veterinary Practitioner. 13: 173-174.

11 Clarke R.E. 1989. Perineal herniorrhaphy in the dog using polypropylene mesh. Australian Veterinary Practitioner. 19: 8-14.

12 Dias M.M., Lhullier F.L.R., Velhos F.A., Oliveira A.P. \& Roehe P.M. 2006. Determinação do cortisol sérico em terneiras aberdeen angus no desmame precoce e tradicional e seus desempenhos reprodutivos até o primeiro parto. Acta Scientiae Veterinariae. 34: 301-306.

13 Fox S.M., Mellor D.J., Laworo C.R.O., Hodge H. \& Firthet E.C. 1998. Changes in plasma cortisol concentrations in bitches in response to different combinations of halothane and butorphanol, with or without ovariohysterectomy. Research in Veterinary Science. 65: 125-133.

14 Grauer G.F., Twedt D.C. \& Mero K.N. 1983. Evolution of laparoscopic for obtaining renal biopsy specimens from dogs and cats. Journal of the American Veterinary Medical Association. 183: 677-679.

15 Guimarães S.M., Oliva V.N.L.S., Maia C.A.A., Ciarlini L.D.R.P., Perri S.H.V., Silva A.R.S., Oliveira D.B. \& Vivian M.C.R. 2007. Correlação de diferentes períodos de jejum com níveis séricos de cortisol, glicemia plasmática, estado clínico e equilíbrio ácido-base em cães submetidos à anestesia geral inalatória. Brazilian Journal of Veterinary Research and Animal Science. 44: 96-102.

16 Hansen B.D., Hardie E.M. \& Carroll G.S. 1997. Physiological measurements after ovariohysterectomy in dogs: what's normal? Applied Animal Behavior Science. 51: 101-109. 
17 Hancock R.B., Lanz O., Waldron D.R., Duncan R.B., Richard V., Broadstone R.V. \& Hendrix P.K. 2005. Comparison of postoperative pain after ovariohysterectomy by harmonic scalpel-assisted laparoscopy compared with median celiotomy and ligation in dogs. Veterinary Surgery. 34: 273-282.

18 Hardie R.J., Flanders J.A., Schmidt P., Credille K.M., Pedrick T.P. \& Short C.E. 1996. Biomechanical and histological evaluation of a laparoscopic stapled gastropexy technique in dogs. Veterinary Surgery. 25: 127-133.

19 Hennessy M.B., Davis H.N., Williams M.T., Mellott C. \& Douglas C.W. 1997. Plasma cortisol levels at a county animal shelter. Physiology \& Behavior. 62: 485-490.

20 Hydbring-Sandberg E., von Walter L.W., Höglund K., Svartberg K., Swenson L. \& Forkman B. 2004. Physiological reactions to fear provocation in dogs, Journal of Endocrinology. 180: 439-448.

21 Inoue T., Ko J.C., Mandsager R.E., Payton M.E., Galloway D.S. \& Lange D.N. 2006. Efficacy and Safety of Preoperative Etodolac and Butorphanol Administration in Dogs Undergoing Ovariohysterectomy. Journal of the American Animal Hospital Association. 42: 178-188.

22 Ishizuka B., Kuribayashi Y., Kobayashi Y., Hamada N., Abe Y., Amemiya A., Aoki T. \& Satoh T. 2000. Stress responses during laparoscopy with $\mathrm{CO} 2$ insufflation and with mechanical elevation of the abdominal wall. The Journal of the American Association of Gynecologic Laparoscopists. 7: 363-371.

23 Ko J.C., Mandsanger R.E. Lange D.N. \& Fox S.M. 2000. Cardiorespiratory responses and plasma cortisol concentrations in dogs treated with medetomidine before undergoing ovariohysterectomy. Journal of the American Veterinary Medical Association. 217: 509-514.

24 Lacy A.M., Garcia-Valdecasas J.C., Piquè J.M., Delgado S., Campo E., Bordas J.M., Taurá P., Grande L., Fuster J., Pacheco J.L. \& Visa J. 1995. Short-term outcome analysis of a randomized study comparing laparoscopic vs open colectomy for colon cancer. Surgical Endoscopy. 9: 1101-1105.

25 Lettow E. 1972. Laparoscopic examinations in liver diseases in dogs. Veterinary Medicine Review. 2: $159-167$.

26 Majeed A.W., Troy G., Nicholl J.P., Smythe A., Reed M.W.R., Stoddard C.J., Peacock J. \& Johnson A.G. 1996. Randomized prospective single-blind comparison of laparoscopic versus small-incision cholecystectomy. Lancet. 347: 989-994.

27 Marcovich R., Williams A.L \& Seifman B.D. 2001. A canine model to assess the biochemical stress response to laparoscopic and open surgery. Journal of Endocrinology. 15: 1005-1008.

28 Peña F.J., Anel L., Domíngues B., Alegre B., Alvarez M., Celorrio I. \& Anel B. 1998. Laparoscopic surgery in a clinical case of seminoma in a cryptorchid dog. The Veterinary Record. 142: 671-672.

29 Raffan P.J. 1993. A new surgical technique for repair of perineal hernias in the dog. Journal of Small Animal Practice. 34 : 13-19.

30 Rawlings C.A., Howert E.W. \& Mahaffey M.B. 2002. Laparoscopic-assisted cystopexy in dogs. American Journal of Veterinary Research. 9: 1226-1231.

31 Remedius A.M. \& Ferguson J. 1996. Minimal invasive surgery: laparoscopic and toracoscopic in the small animals. The Compendium of Continuing Education for the Practicing Veterinarian. 18: 1191-1199.

32 Rudd R.G. \& Hendrickson D.A. 1998. Minimally invasive surgery of the urinary system. In: Freeman L.J. (Ed.). Veterinary Endosurgery. St. Louis: CV Mosby, pp.226-236.

33 Stage J.G., Schultze S., Moller P., Overgaard H., Andersen M., Rebsdorf-Pedersen V.B. \& Nielseni H.J. 1997. Prospective randomised study of laparoscopic versus open colonic resection for adenocarcinoma. British Journal of Surgery. 84: $391-396$.

34 Siegl V.H., Böhm R. \& Ferguson J. 1994. Laparoskopische ovariohysterektomie bei einem hund. Wiener Tierärztliche Monatsschirft. 81: 149-152.

35 Terlouw E.M.C., Schouten W.G.P. \& Ladewig J. 1997. Physiology. In: Appleby M.C. \& Hughes B.O. (Eds). Animal Welfare. Wallingford: CABI Publishing, pp.143-158.

36 Vieira J.G.H., Nakamura O.H. \& Carvalho V.M. 2005. Measurement of free urinary cortisol and cortisone using liquid chromatography associated with tandem mass spectrometry method. Arquivo Brasileiro de Endocrinologia \& Metabologia. 49: 291-298.

37 Vincent I.C. \& Leahy R.A. 1997. Real-time non-invasive measurement of heart rate in working dogs: a technique with potential applications in the objective assessment of welfare problems. The Veterinary Journal. 153: 179-183.

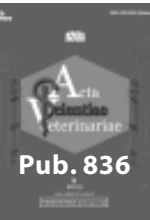

